\title{
Sexual Disorders Seen in Alcohol Dependent Patients
}

\author{
Amitabh Saha ${ }^{1}$, Bikram Dutta ${ }^{2}$, Shahbaz Ali Khan ${ }^{3}$ \\ ${ }^{1}$ Department of Psychiatry, Maharashtra University, Command Hospital, Pune, Maharashtra, India \\ ${ }^{2}$ Department of Psychiatry, UP University, Command Hospital, Lucknow, Uttar Pradesh, India \\ ${ }^{3}$ Department of Psychiatry, Base Hospital, Delhi University, New Delhi, India
}

\section{Email address:}

sahaing@gmail.com (A. Saha)

\section{To cite this article:}

Amitabh Saha, Bikram Dutta, Shahbaz Ali Khan. Sexual Disorders Seen in Alcohol Dependent Patients. American Journal of Psychiatry and Neuroscience. Vol. 3, No. 2, 2015, pp. 30-39. doi: 10.11648/j.ajpn.20150302.13

\begin{abstract}
The chances of developing sexual dysfunctions appear to increase with increasing years of alcohol consumed. Various explanations have been offered for sexual dysfunction in alcoholic patients. Higher level of alcohol intake may result in greater neurotoxic effects. The prevalence of sexual disorders in those people who consume alcohol has been known to occur frequently. The etiology of this dysfunction needs to be considered. This study has focused on the prevalence of sexual dysfunction in chronic alcohol consumers fulfilling the diagnostic criteria for alcohol dependence syndrome.
\end{abstract}

Keywords: Sexual Dysfunction, Erectile Dysfunction, Premature Ejaculation, Psychiatric Morbidity, Orgasmic Dysfunction, Alcohol Dependence

\section{Introduction}

A sexual dysfunction can arise as a result of biological problems, relationship problems, intra psychic conflicts, lack of a proper sexual knowledge, other psychiatric and or Medical disorders, personality, types and disorders, and use of medicines (anti-hypertensive, anti-depressants) or a combination of any or all of these. Sexual dissatisfaction and sexual dysfunction today occur with a frequency that would overwhelm the health services if they all presented for help. Sexual problems, generally believed to have wide prevalence, have been accorded low priority and physicians either show no interest or tend to ignore patient's psychosexual complaints (1).

Proper sexual functioning is one of the most important components of quality of life and of maintaining a satisfying intimate relationship and it provides a sense of physical, psychological \& social wellbeing (2).

Virtually all aspects of the human sexual response are affected by alcohol especially sexual desire and erection (3)

\section{Need for this Study}

In this study we will assess what is the prevalence and different types of sexual dysfunction among freshly diagnosed Alcohol Dependence Syndrome male patients.

\section{Materials and Methods}

Type of study: Cross sectional case control study

Setting: The study was carried out in tertiary care hospital, which is also a teaching institute. Subjects, consecutively evaluated in the de addiction Ward, with a diagnosis of Alcohol dependence Syndrome (ICD-10) during a period of 1 year from May 2012 to April 2013 were recruited for the study.

\subsection{Sample}

Study group: The study was limited to male subjects because the hospital had only male inpatient in de addiction ward facilities. The age of these patients was between 20-60 years because the patients getting admitted were in this age group. A total of 50 alcohol dependent male inpatients were recruited for this study.

\subsection{Inclusion Criteria}

a. All cases meeting the criteria for Alcohol Dependence Syndrome

b. Between 20-60 years of age

c. Married or having a regular sexual partner

\subsection{Exclusion Criteria}

a. Clinically assessed history of primary sexual 
dysfunction [prior to initiation of alcohol use]

b. Co-morbid physical disorders: e.g. diabetes mellitus, thyroid disorders, other systemic illnesses, anatomical or structural perineal, genitourinary lesions and neurological or spinal cord lesions.

c. Co-morbid psychiatric disorders: schizophrenia, delusional disorder, anxiety disorders and mood disorders.

d. Substance use other than alcohol and tobacco.

\subsection{Control Group}

The controls were made up age and education matched healthy individuals not dependent on alcohol. After applying same exclusion criteria as that of study group, controls were recruited in the study.

\subsection{Materials}

Specially designed semi-structured Performa to collect demographical data was used.

\subsection{Scales Used}

1. Alcohol Use Disorder Identification Test (AUDIT)

2. Hamilton Rating Scale for Depression (HAM-D)

3. Hamilton Rating Scale for Anxiety (HAM-A)

4. Brief Sexual Function Inventory (BSFI) (Appendix B)

5. International Index of Erectile Function (IIEF) (Appendix C)

6. Index of Premature Ejaculation (IPE) (Appendix D)

\subsection{Methodology}

Baseline demographic data of cases along with relevant investigations were entered in semi-structured preformed in first week of admission. Alcohol use further assessed with AUDIT (Alcohol Use Disorder Identification Test).

To rule out organic causes of sexual dysfunction, appropriate tests (biochemical, endocrinological, and radiological) were carried out. These comprised of complete haemogram To Rule out Anemia, Blood-Sugar Profiles, Hepatic And Renal Function Tests, (Rule Out Infections Of urinary tract) and VDRL, testosterone levels, and USG abdomen. Testosterone analysis was done using radioimmunoassay.

All the above subjects were assessed for the prevalence of one or more sexual dysfunction using Brief Sexual Function Inventory (BSFI). In addition, presence of sexual dysfunction in two commonly reported dysfunctional domains, inadequate erection and premature ejaculation, was further elaborated by Premature Ejaculation (IPE) to all subjects in the study and control groups.

All patients were assessed by Hamilton Rating Scale for Depression (HAM-D)(4) \& Hamilton Rating Scale for Anxiety (HAM-A) (5).The ratings on all the above mentioned scales were sought after two weeks of inpatient stay after the period of detoxification with benzodiazepine was over. Scales like AUDIT have been used in studies earlier dealing with alcohol use and other medical conditions.(6)

Similarly, demographic data was recorded from individuals of matched control group. Individuals from control group were assessed for sexual functioning with the BSFI, IIEF and IPE. Data was statistically analyzed with suitable statistical method.

\subsection{Statistical Analysis}

The data was analyzed using Statistical Package for Social Sciences (SPSS) version 20.

\subsection{Description of Scales Used}

\subsubsection{Brief Sexual Function Inventory (BSFI)}

It is a brief self-report inventory developed by $\mathrm{Mp}$ O'Leary and collaborators (7) to measure the current sexual function of males. The scale has 11 items, scored on a 5-point like type scale that covers sex drive, erectile capacity, ejaculatory function and satisfaction with sexual performance. For the present study the cut off score for each domain was chosen to be the point from where dysfunction begins. The cut off scores for sexual drive was $<7$, for erection it was taken as $<10$ and for overall satisfaction it was taken as $<4$.

\subsubsection{IIEF (International Index of Erectile Function)}

The IIEF (8) addresses the relevant domains of male sexual function (that is, erectile function, orgasmic function, sexual desire, intercourse satisfaction, and overall satisfaction), is psychometrically sound, and has been linguistically validated in multiple languages. .Dimension (s) covered by the questionnaire:

Erectile function (6items)

Orgasmic function (2 items)

Sexual desire (2 items)

Intercourse satisfaction ( 3 items)

Overall satisfaction ( 2 items)

The scoring of the IIEF segregates the values of the domain of erectile function according to the severity.

For erectile function

1-10: Severe Erectile Dysfunction

11-16: Moderate Dysfunction

17-21: mild to moderate Dysfunction

22-25: mild Dysfunction

26-30: No Dysfunction

\subsubsection{The Index of Premature Ejaculation (IPE)}

It is a self-report questionnaire developed to address issues associated with ejaculating prematurely, The IPE addresses three aspects: sexual satisfaction, control over ejaculation, and distress about the condition. The cut off scores for sexual satisfaction it was less than 17 , for problems of control of ejaculation it was less than 17 and for distress related to ejaculatory difficulties it was less than 9. (9)

\subsubsection{Alcohol Use Disorders Identification Test (AUDIT) Questionnaire}

The AUDIT questionnaire 144 is a screening test specifically designed for international use. The questionnaire 
helps the practitioner identify whether the person has hazardous drinking, harmful drinking, or alcohol dependence, It is a 10 -item questionnaire; it takes about two minutes to complete; each question is scored from 0 to 4 ; and the maximum score is 40 . A total score of 8 or more on the questionnaire suggests that the person has a pattern of hazardous or harmful alcohol consumption. As a general guide, if a score is 13 or more, it is likely that the person is alcohol dependent.

\section{Results and Observations}

Table 1. Demographic profile of study and control group.

\begin{tabular}{llll}
\hline & Minimum & Maximum & Mean \\
\hline Age of study group (years) $\mathrm{n}=50$ & 26.00 & 49.00 & 39.000 \\
Education of study group (years) $\mathrm{n}=50$ & 5.00 & 15.00 & 10.4600 \\
Age of control group (years) $\mathrm{n}=50$ & 27.00 & 49.00 & 2.05 \\
Education of control group (years) $\mathrm{n}=50$ & 7.00 & 15.00 & 37.3800 \\
\hline
\end{tabular}

Table 1 shows the age distribution of the participants as well as the educational status of the participants in the study group and the control group.

Table 2. Comparison of demographic data of study and control group using the Mann Whitney Test.

\begin{tabular}{llll}
\hline & $\begin{array}{l}\text { Study group mean } \\
\text { score }(\mathbf{N}=\mathbf{5 0})\end{array}$ & $\begin{array}{l}\text { Control group mean } \\
\text { score }(\mathbf{N}=\mathbf{5 0})\end{array}$ & P value \\
\hline $\begin{array}{l}\text { Age (years) } \\
\begin{array}{l}\text { Education } \\
\text { (years) }\end{array}\end{array}$ & 39.3000 & 37.3800 & 0.118 \\
\hline
\end{tabular}

Table 2 compares the age in years and the educational status in years of the two groups. Statistical analysis shows that there is no statistical difference between the age and the educational status of the two groups.

Table 3. Prevalence of Sexual Dysfunction as per Brief Sexual Function Inventory (BSFI).

\begin{tabular}{lll}
\hline Domain & Study group $(\mathbf{N}=\mathbf{5 0})$ & Control group $(=\mathbf{5 0})$ \\
\hline No dysfunction & $14(28 \%)$ & $35(70 \%)$ \\
Low Sexual Drive & $28(56 \%)$ & $6(12 \%)$ \\
Erectile dysfunction & $23(46 \%)$ & $5(10 \%)$ \\
Ejaculation Problem & $16(32 \%)$ & $8(16 \%)$ \\
Problem Assessment & $26(52 \%)$ & $14(28 \%)$ \\
Poor Overall & $03(60 \%)$ & $15(30 \%)$ \\
Satisfaction &
\end{tabular}

Table 3 depicts the prevalence rates of the different domains of sexual dysfunction as per the Brief Sexual Function Inventory (BSFI) of the cases compared to the controls.14 (28\%) subjects of the study group and $35(70 \%)$ subjects of the control group did not report any sexual dysfunction on any of the domains.

On BSFI, the most common sexual dysfunction seen in male patients was low sexual Drive, being present in 28 $(56 \%)$ subjects of study group compared to $6(12 \%)$ subjects in the control group.

Erectile dysfunction and Ejaculation problem were seen in $23(46 \%)$ and $16(32 \%)$ subjects respectively in the study group, and $5(10 \%)$ and $8(16 \%)$ subjects respectively in the control group. Around 26 (52\%) subjects of study group had a problem with sexual life compared to 14 (28\%) subjects of control group. $30(60 \%)$ subjects of study group were overall dissatisfied with their sexual life compared to $15(30 \%)$ subjects of control group.
Table 4. Prevalence of Sexual Dysfunction as per International Index of

\begin{tabular}{lll}
\hline DOMAIN & Study group $(\mathbf{N}=50)$ & Control group (=50) \\
\hline No dysfunction & $13(26 \%)$ & $40(80 \%)$ \\
Erectile dysfunction & $24(48 \%)$ & $8(16 \%)$ \\
Intercourse & $25(50 \%)$ & $6(12 \%)$ \\
dissatisfaction & $7(14 \%)$ & $2(4 \%)$ \\
Orgasmic dysfunction & $29(58 \%)$ & $9(18 \%)$ \\
Decreased sexual desire & $24(68 \%)$ & $7(14 \%)$ \\
Overall dissatisfaction & $34(6)$ & \\
\hline
\end{tabular}

Table 4 depicts the prevalence rates of the different domains of sexual dysfunction as per the International Index of Erectile Function of the subjects of the study group competed to the control group. $13(26 \%)$ subjects of the study group and $40(80 \%)$ subjects of the control group did not report any dysfunction on any of the domains.

On IIEF, $24(48 \%)$ subjects of the study group had problems of erectile function compared to $8(16 \%)$ of the controls. $25(50 \%)$ of the subjects of the domains. dissatisfaction with intercourse and orgasmic dysfunction compared with $12 \%$ and $4 \%$ of the controls respectively. Among the study group 29 (58\%) had decreased sexual desire and there was a decrease in overall satisfaction of 26 $(52 \%)$ subjects. In the control group decreased sexual desire and decrease in overall satisfaction were seen in $9(18 \%)$ and 7 (14\%) subjects respectively.

Table 5. Distribution of subjects of study and control group according to severity of erectile dysfunction in IIEF.

\begin{tabular}{lll}
\hline & Study group $(\mathbf{N}=\mathbf{5 0})$ & Control group $(=\mathbf{5 0})$ \\
\hline No dysfunction & 26 & 42 \\
Mild dysfunction & 7 & 6 \\
$\begin{array}{l}\text { Mild to moderate } \\
\text { dysfunction }\end{array}$ & 13 & 2 \\
Moderate dysfunction & 4 & 0 \\
Severe dysfunction & 0 & 0 \\
\hline
\end{tabular}

Table 5 depicts the distribution of subject of the study group and control group according to severity of erectile dysfunction as per the IIEF. Among the subjects of study group having erectile dysfunction (24), 7 had mild dysfunction, 13 had mild to moderate dysfunction, 4 had moderate dysfunction. Erectile Function (IIEF). 
Table 6. Prevalence of sexual dysfunction as per Index of Premature Ejaculation (IPE).

\begin{tabular}{lll}
\hline DOMAIN & Study group $(\mathbf{N}=\mathbf{5 0})$ & Control group $(=\mathbf{5 0})$ \\
\hline No dysfunction & $16(32 \%)$ & $4080 \%)$ \\
Sexual satisfaction & $32(64 \%)$ & $4(8 \%)$ \\
Control & $18(36 \%)$ & $5(10 \%)$ \\
Distress & $21(42 \%)$ & $9(18 \%)$ \\
\hline
\end{tabular}

Table 6 shows dysfunction of the domains of the Index of Premature Ejaculation. Among the study group 32 (64\%) had a decrease in sexual satisfaction, $18(36 \%)$ had decreased control over their ejaculation and 21 (42) \% subjects expressed distress about their condition of premature ejaculation. Among the control group $4(8 \%)$ had decrease in satisfaction with sexual life, $5(10 \%)$ had decreased control over their ejaculation and $9(18 \%)$ expressed distress about their ejaculatory function.
Table 7. Mean domain scores for BSFI of subjects of study group compared with Control group using the Mann Whitney Test.

\begin{tabular}{llll}
\hline Domain & $\begin{array}{l}\text { Study group mean } \\
\text { score }(\mathbf{N}=\mathbf{5 0})\end{array}$ & $\begin{array}{l}\text { Control group mean } \\
\text { score }(\mathbf{N}=\mathbf{5 0})\end{array}$ & P value \\
\hline Sexual drive & 6.1600 & 7.5400 & $<0.01$ \\
Erection & 9.1200 & 11.3000 & $<0.01$ \\
Ejaculation & 7.0000 & 7.5800 & $<0.01$ \\
$\begin{array}{l}\text { Problem } \\
\text { assessment }\end{array}$ & 9.1800 & 11.1800 & $<0.01$ \\
$\begin{array}{l}\text { Overall } \\
\text { satisfaction }\end{array}$ & 3.0600 & 3.7000 & $<0.01$ \\
\hline
\end{tabular}

Table 7 shows a comparison of the mean scores of the domains of sexual function of the BSFI between the study and the control groups. On statistical analysis by the Mann Whitney test there was a significant difference $(p<0.01)$ among all the domains between the study group and the control group.

Table 8. Mean domain scores for IIEF of study group compared with control Group using the Mann Whitney Test.

\begin{tabular}{llll}
\hline Domain & Study group mean score $(\mathbf{N}=\mathbf{5 0})$ & Control group mean score $(\mathbf{N}=\mathbf{5 0})$ & P value \\
\hline Erectile function & 23.7800 & 28.4200 & $<0.01$ \\
Intercourse satisfaction & 12.0400 & 14.2400 & $<0.01$ \\
Orgasmic function & 9.1200 & 9.7800 & $<0.01$ \\
Sexual desire & 7.6200 & 9.5200 & $<0.01$ \\
Overall satisfaction & 7.5200 & 9.5400 & $<0.01$ \\
\hline
\end{tabular}

Table 8 shows a comparison of the mean scores of the domains of sexual function of the IIEF between the study and the control groups. On statistical analysis by the Mann
Whitney test there was a significant difference $(\mathrm{p}<0.01)$ among all the domains between the study and the control groups.

Table 9. Mean domain scores for IPE of study group compared with control group Using the Mann Whitney Test.

\begin{tabular}{lll}
\hline Domain & Study group mean score $(\mathbf{N}=\mathbf{5 0})$ & Control group mean score $(\mathbf{N}=\mathbf{5 0})$ \\
\hline Sexual Satisfaction & 15.2000 & 19.1400 \\
Control & 17.1400 & 18.9600 \\
Distress & 8.2600 & 9.5200 \\
\hline
\end{tabular}

Table 9 shows a comparison of the mean scores of the domains of sexual function of the IPE between the study and the control group, on statistical analysis by the Mann
Whitney test there was a significant difference $(p<0.01)$ among all the domains between the study and the control groups.

Table 10. Correlation of domains of BSFI with other variables of the study group using Spearman's Rho correlation.

\begin{tabular}{llllll}
\hline & Sexual drive (r) & Erection $(\mathbf{r})$ & Ejaculation $(\mathbf{r})$ & Problem assessment (r) & Overall satisfaction (r) \\
\hline Years of tobacco consumption & -.215 & .072 & .199 & .096 & .098 \\
(smoking or chewing) & -.058 & .115 & .241 & .130 & .146 \\
Presenting age & -.541 & -0.519 & -0.581 & -0.619 & -0.558 \\
Years of drinking & -.008 & .039 & -.109 & -.026 & .019 \\
AUDIT & -.099 & -.132 & -.175 & 0.23 & -.194 \\
Testosterone & & & & \\
\hline
\end{tabular}

*Correlation is significant at the 0.01 level (2-tailed)

Table 10 shows the study of the correlation between the variables of the study group and the domains of sexual function of the BSFI. A significant negative correlation was seen between years of drinking and impairment of all the domains of the BSFI. The other variables i.e. years of tobacco consumption (smoking or chewing), presenting age i.e. age at being diagnosed as Alcohol Dependence Syndrome (ADS), AUDIT score, Serum testosterone levels had no significant correlation with the domains of the BSFI 
Table 11. Correlation of domains of IIEF with other variables of the study group using Spearman's Rho correlation.

\begin{tabular}{llllll}
\hline & Sexual drive $(r)$ & Erection $(\mathbf{r})$ & Ejaculation $(\mathbf{r})$ & Problem assessment (r) & Overall satisfaction (r) \\
\hline Years of tobacco consumption & .042 & -.024 & .141 & -.197 & -.078 \\
(smoking or chewing) & .200 & .020 & -.001 & -.113 & -.161 \\
Age at being diagnosed as ADS &. $.493^{*}$ & -.254 & $-.579^{*}$ & $-.456^{*}$ & $-.458^{*}$ \\
Years of drinking & .179 & .056 & -.150 & .221 & .068 \\
AUDIT score & -0.84 & .079 & -.026 & -.022 & .020 \\
Serum Testosterone levels & &
\end{tabular}

*Correlation is significant at the 0.01 level (2-tailed)

Table 11 shows the study of the correlation between the variables of the study group and the domains of sexual function of the IIEF. A significant negative correlation was seen between years of drinking and impairment of the domains of the IIEF except for the domain of intercourse satisfaction. The other variables i.e. years of tobacco consumption (smoking or chewing), age at being diagnosed as ADS, AUDIT score, serum testosterone levels had no significant correlation with the domains of the IIEF.

Table 12. Correlation of domains of IPE with other variables of the study group using Spearman's Rho correlation.

\begin{tabular}{llll}
\hline & Sexual satisfaction (r) & Control ( r) & Distress (r) \\
\hline Years of tobacco consumption (smoking or chewing) & -.026 & .228 & -.075 \\
Presenting age & -.069 & .169 & .085 \\
Years of drinking & $-.452 *$ & $-.589 *$ & $-.414^{*}$ \\
AUDIT & .055 & -.257 & -.123 \\
Testosterone & -.058 & -.094 & -.130 \\
\hline
\end{tabular}

*Correlation is significant at the 0.01 level (2-tailed)

Table 12 shows the study of the correlation between the variables of the study group and the domains of sexual function of the IPE. A significant negative correlation was seen between years of drinking and impairment of all the domains of the IPE. The other variables i.e. years of tobacco consumption (smoking or chewing), age at being diagnosed as Alcohol Dependence Syndrome, AUDIT score, Serum testosterone levels had no significant correlation with the domains of the IPE

Table 13. Testosterone values of study group.

\begin{tabular}{llll}
\hline Study group $\mathbf{N}=\mathbf{5 0}$ & Minimum & Maximum & Mean \\
\hline Serum testosterone $(\mathrm{ng} / \mathrm{dl})$ & 270.00 & 1480.00 & 513.6400 \\
\hline
\end{tabular}

Table 13 shows the mean values of the serum testosterone levels of the study group which is $513.64 \mathrm{ng} / \mathrm{dl}$. None of the participants had decreased serum testosterone levels (Normal range $270-1070 \mathrm{ng} / \mathrm{dl}$ ). One participant had increased serum testosterone level of $1480 \mathrm{ng} / \mathrm{dl}$.

Table 14. HAM A and HAM D scores of study group.

\begin{tabular}{llllll}
\hline & N & Minimum & Maximum & Mean & $\begin{array}{l}\text { Std. } \\
\text { Deviation }\end{array}$ \\
\hline HAM D & 50 & 0 & 6 & 2.26 & 1.75 \\
HAM A & 50 & 0 & 5 & 1.9400 & 1.33 \\
\hline
\end{tabular}

Table 14 depicts the scores of the study group on HAM D and HAM A rating scales. It signifying that none of the participants of the study group were significantly depressed or anxious as their scores were well below the cut off scores for presence of depression or anxiety.

\section{Discussion}

\subsection{Sociodemographic Data}

Alcoholic Dependency syndrome is known to be associated with sexual dysfunction.

In this study, 50 patients of alcohol Dependence syndrome were included of which all were males, were married for a period of at least 1 year. In this cross sectional, noninterventional, case control study, 50 patients of alcoholic dependency syndrome included were evaluated for the presence of sexual dysfunction on the basis of three selfrelated scales; the BSFI, IIEF, the IPE. Their scores were compared to those of a matched group of healthy, nonalcohol dependent controls. As seen in Table 1, the 50 male patients of the study group were in the age group of 26-49 years, with a mean age of 39.3 years. The control group were in the age group of 27-49 years and their mean was 37.4 years. There was no statistically significant difference in the age of the two groups as seen in Table 2. This eliminates any age bias that could be present for impaired sexual functioning while comparing the 2 groups.

There was also no statistical significance in the educational status of the 2 groups as seen in table 2. The educational status of the study group had a mean of 10.46 years of education while the control group educational status had a mean of 10.66 years of education.

\subsection{Assessment by the Brief Sexual Function Inventory (BSFI)}

The brief sexual function inventory assesses for 
functioning in five domains which are Sexual drive, problems related to erection, ejaculation problems, problem assessment and overall satisfaction. Among the study group as seen in table 3, $28(56 \%)$ participants had scores of decreased sex drives, 23(46\%) had problems related to Erectile function and $16(32 \%)$ had problem related to ejaculation (table 3). A total of 26(52\%) participants reported some problem with their sexual functioning and $30(60 \%)$ participants were not fully satisfied with their sexual life. As seen in Table 3, 14 subjects of the study group did not report any sexual dysfunction on the BSFI i.e. 36 patients (72\%) had reported some dysfunction on at least one of the Domains. 35 controls did not report any dysfunction (Table 3) on any of the domains i.e.15 (30\%) of the controls reported some of the dysfunction on at least one of the domains.

Studies on subjects who are long term alcohol users have shown variable results for the prevalence of sexual dysfunction. Studies have revealed that of 97 males in patients admitted for the treatment of alcoholism, $71 \%$ suffered from sexual dysfunction for a period of more than 12 months prior to admission to a hospital (10). Vivek Benegal in their study on 100 alcohol dependent patients found that 72 of the 100 subjects reported one or more sexual dysfunction (11). Mandell et al interviewed 44 alcoholic men and found that $84 \%$ reported some sexual dysfunction related to alcohol abuse (12). The findings of this study were in concordance with these results, thus indicating that Sexual dysfunction is common in patients of Alcohol Dependence Syndrome. Schiavi et al failed to find any difference in sexual dysfunction in alcoholics abstinent for 2-3 months in comparison with a non-alcoholic control group, speculating that Alcohol -induced sexual dysfunction was reversible with abstinence (13).

\subsubsection{Sex Drive}

Among the study group 28 participants (56\%) had scores of decreased sex drive compared to $6(12 \%)$ subjects of the control group (Table 3). On BSFI the mean score of sexual drive domain of the study group i.e. alcohol dependents was 6.16 while the mean score of the controls was 7.54. The difference between the two was statistically significant ( $p$ $<0.01$ ) (table 7).Studies showing significant decrease in sexual drive included those by Vivek Benegal (12).Jensen reported that $63 \%$ of married alcoholic men and $10 \%$ of controls had sexual dysfunction, especially lack of sexual of desire. In his study on male Alcoholic inpatients (14) Vijayasenan had noticed diminished sexual desire in $58 \%$ of the Patients.

Gumus et al reported in their study that $24.4 \%$ chronic alcoholics had decrease in Sexual desire when they used alcohol. This was not statistically significant to the Control group.

Ronald W. Lewis et al in their review of literature from around the world in the general population found that in five different reports, the prevalence of desire/interest dysfunction, without any pronounced aging effect, of about $25 \%$ was reported for man of the age group of 16-59 and 40-80 years.
The prevalence rate for decreased sexual desire in our study group was higher than the above findings in the general population thus implying that decreased sexual desire is common in alcohol Dependence Syndrome.

\subsubsection{Erection}

Episodic erectile failure in alcoholic men is fairly routine, found to be significantly higher in men consuming more than three standard units of alcohol. In our study on assessment of the domain of erection we found that 23 patients $(46 \%)$ had reported problems related to erectile functioning compared to $5(10 \%)$ subjects in the control group (Table 3$)$. On BSFI the mean score of erectile function domain of the study group i.e. alcohol dependents was 9.12 while the mean score of the control was11.3. The difference between the two was statistically significant $(\mathrm{p}<0.01)$ (Table 3$)$.

Ronald W. Lewis in their view of literature on the prevalence of erectile dysfunction in the general population found that below the age of 40 years, prevalence for erectile dysfunction was $1-10 \%$ and in the decade from $40-49$, the prevalence ranges from $2 \%$ to $9 \%$ to as high as $15 \%(15)$. Our findings are higher compared to his review thus implying that the prevalence of erectile dysfunction in patients of Alcohol Dependence Syndrome is much more than that in the general population. Various other studies have reported increased prevalence of erectile dysfunction in subjects with Alcohol Dependence Syndrome. In his study Whaly had reported that $54 \%$ of hospitalized alcoholic men and $24 \%$ of healthy controls had erectile impotence. Mandell et.al. interviewed 44 alcoholic men and found that 59\% experienced erectile dysfunction during periods of heavy drinking. Van Thiel and Lester reported that $61 \%$ of patients dependent on alcohol reported sexual dysfunction, the most common being erectile dysfunction (16). Bijil Simon Arackal and Vivek Benegal had studied 100 subjects of Alcohol Dependence Syndrome and in their concordance with the above findings implying that erectile disturbances are present in around half of the subjects of Alcohol Dependence Syndrome (18).

There are few studies quoting lesser prevalence rates for erectile dysfunction. Vijayasenen reported that of 97 male in patients admitted for the treatment of alcoholism, there was disturbance of erectile impotence in $16 \%$ of the subjects with a71\% of sexual dysfunction overall (10). Eva-Maria Fahrner had assessed for sexual Dysfunction over the past 6 months in alcoholics and has reported a $22 \%$ prevalence of erectile dysfunction. The lower prevalence rates in these studies could be due to the different sample size and different methodology used for assessing erectile functioning (17).

\subsubsection{Ejaculation}

In our study 16 subjects (32\%) reported problems with ejaculatory function in the study Group compared to $8(16 \%)$ subjects in the control group (Table 3). On BSFI the mean Score of ejaculatory function domain of the study group was 7.0 group while the mean score of the control was 7.58 . The difference between the two was statistically significant $(p<0.01)$ (Table 7). The findings imply that ejaculatory function too is affected in subjects with Alcohol Dependence 
Syndrome. Premature ejaculation was assessed by various studies and have reported rates from about $20 \%-30 \%$ of the male population across all age groups.

Concordance prevalence rates to our study was found by Bijil Simon Arackal and Vivek Benegal (18) on their study on alcoholics where premature ejaculation was reported by 36 out of 96(37.5) subjects (18). Also Mandell et. Al. in their review of 44 volunteers in a county alcoholism program reported $48 \%$ prevalence of ejaculatory incompetence.

Eva-Maria Fahrner on chronic Alcoholics found prevalence of premature ejaculation to be $18 \%$.Vijaysenen in his study of 97 patients found premature ejaculation in 4\%. This possibly could be due to different methodology and sample structure or the different rate of reporting of perceived dysfunction by the patients due to inadequate psychological mindedness. Studies have quoted the presence of premature ejaculation in alcoholics and that secondary premature ejaculation may sometimes be found in association with alcohol-related peripheral neuropathy.

\subsubsection{Problem Assessment and Overall Satisfaction}

The domain of problem assessment is a comprehensive score relating to question on how subjects perceived problems related to sexual drive, erection and ejaculation. In Our study 26 subjects (52\%) of the study group had reported problem in their sex life compared to $14(28 \%)$ subjects in the control group (Table3).On BSFI the mean score of problem assessment domain of the study group was 9.18 while the mean score of the controls was 11.18. The difference between the two was statistically significant $(\mathrm{p}<0.01)$ (Table 7).

$30(60 \%)$ of the subjects of the study group had decreased overall satisfaction with their Sex life compared to $15(30 \%)$ of subjects of the control group (Table 3). On BSFI the mean score of overall satisfaction domain of the study group was 3.08 while the mean score of the control was 3.7. The difference between the two was statistically significant $(\mathrm{p}<0.01)$ (Table 7).

These results show that there is relative consistency between the 2 domains that as the subjects perceive problems related to their sexual functioning their overall satisfaction of their sex life decreases. These scores were statistically significant compared to those of controls thus implying that alcohol increases problems related to sexual functioning and decreases overall satisfaction of sex life.

\subsection{Assessment Based on the International Index of Erectile Function (IIEF)}

The IIEF addresses the relevant domains of male sexual function (that is, erectile function, orgasmic function, sexual desire, intercourse satisfaction, and overall satisfaction). As seen in Table 4,13 subjects of the study group did not report any Sexual dysfunction on the IIEF i.e.37 patients(74\%) had reported some dysfunction on at least one of the domains. 40 controls did not report any dysfunction(Table 4) on any of the domains i.e. $10(20 \%)$ of the controls reported some dysfunction on at least one of the domains.

\subsubsection{Erectile Function}

On further assessing for erectile function using the IIEF in this study group we found that 24 subjects (48\%) reported problems related to erectile functioning (Table 4) which is similar to the results obtained on assessment by the BSFI and in concordance with earlier mentioned studies. In the control group $8(16 \%)$ participants reported dysfunction (Table 4). On the IIEF for the domain of the erectile function the mean score of the study Group was 23.78 while that of the control group was 28.42 . This was statistically significant compared to the control group $(\mathrm{p}<0.01)$ (Table 8$)$.

For the severity of erectile dysfunction assessed by the IIEF among the study group, 7 (14\%) had reported mild dysfunction. $13(26 \%)$ had reported mild to moderate dysfunction and 4(8\%) had reported moderate dysfunction; and none of the participants had reported severe dysfunction (Table 5). The findings of this study suggests that erectile dysfunction due to alcohol consumption is mainly of mild to moderate severity.

\subsubsection{Intercourse Satisfaction}

The IIEF also assessed for other domains like intercourse satisfaction, or orgasmic function, sexual desire and overall satisfaction. On assessment on the domain of intercourse satisfaction $25(50 \%)$ subjects reported that they were not satisfied with their sexual functioning during intercourse compared to $6(12 \%)$ subjects of the control group not being satisfied with their sexual functioning during intercourse (Table 4). The mean score of the subjects of the study group were12.04 while the mean score in the control group was 14.24. The difference between the two groups was statistically significant $(\mathrm{p}<0.01)$. (Table8).

\subsubsection{Orgasmic Function}

$7(14 \%)$ subjects of the study group reputed disturbances of orgasmic function compared to $2(4 \%)$ subjects of the control group (Table4). The mean score of the study group on the domain of orgasmic function was 9012 and that of the control group was 9.78. The difference between the two groups was statistically significant $(\mathrm{p}<0.01)$. (Table 8)Simon Arackal and Vivek Benegal (18) in their study found that fourteen subject $(14.58 \%)$ had a lack of pleasure at the time of ejaculation (anorgasmia) and $10(10.41 \%)$ had inhibited or delayed ejaculation.

\subsubsection{Sexual Desire and Overall Satisfaction}

In this study $29(58 \%)$ subjects of the study group and 9 $(18 \%)$ subjects of the control group reported decreased sexual desire (Table 4). The mean score of the study group on the domain of sexual desire was 7062 and that of the control group was 90520 . The difference between the two groups was statistically significant $(\mathrm{p}<0.01)$. (Table 8$)$ this finding again correlated with the findings of decrease sexual drive according to the BSFI. 34(68\%) participants of the study group had decreased overall satisfaction compared to 7 (14\%) subjects of the control group (Table 4). The mean score of the study group on the domain of overall satisfaction was 7.52 and that of the control group was 9.54. The difference 
between the two groups was statistically significant $(\mathrm{p}<0.01)$ (Table 8). These results are in concordance with the findings related to $\mathrm{BSFI}$.

\subsection{Assessment Based on the Index of Premature Ejaculation (IPE)}

The IPE addresses issues associated with ejaculating prematurely. The IPE addresses three aspects: sexual satisfaction, control over ejaculation, and distress about the condition. In the study group $32(64 \%)$ subjects reported a decrease in sexual satisfaction overall while in the control group $4(8 \%)$ subjects reported a decrease in sexual satisfaction (Table 6). The mean score of the study group on the domain of sexual satisfaction was 1502 and that of the control group was 19.14. The difference between the two groups was statistically significant $(\mathrm{p}<0.01)$. (Table 9) This prevalence rate was similar to that reported for decreased overall satisfaction as per the BSFI18 (36\%) subjects of the study group and $5(10 \%)$ subjects of the control group complained of difficulty in control of their ejaculation (Table 6 ). The mean score of the study group on the domain of ejaculatory control was 17.14 and that of the control group was 18.96. The difference between the two groups was statistically significant ( $<0.01)$, (Table 9) $21(42 \%)$ subjects of the study group reported distress about their premature ejaculation while in the control group $9(18 \%)$ subjects did so (Table 6). The mean score of the study group on the domain of distress due to premature ejaculation was 8.26 and that of the control group was 9.52. The difference between the two groups was statistically significant $(p<0.01)$. (Table 9). The scores on all the domains of the IPE were statistically significant when compared to the control group (table 9). This implies the premature ejaculation is common in subjects with Alcohol Dependence Syndrome. These subjects have difficulty controlling their ejaculation and have significant distress related to it. The prevalence rates of problems due to ejaculatory function on the BSFI and on the IPE are slightly different which may be due to inter vast variability, minor differences in the questions and the different cut off scores selected as per scale.

\subsection{Correlations of Domains of Sexual Functioning with Variables of Study Group}

The different domains of sexual functioning was correlated with years of tobacco consumption (smoking or chewing), current age of diagnosis of subjects with alcohol dependence syndrome, the number of years the subject has been consuming alcohol, AUDIT score and serum testosterone levels. Table 10 shows the correlation of the different domains of sexual function of the BSFI, Table 11 shows the correlation of the different domains of sexual function of the IIEF and Table 12 shows the correlation of the different domains of sexual function of the IPE with years of tobacco consumption (smoking or chewing), current age of diagnosis of subjects with alcohol dependence syndrome, the number of years the subject has been consuming alcohol, AUDIT score and serum testosterone levels.

Only years of alcohol consumption prior to current assessment had a significant correlation with almost all the domains of sexual functioning except on the domain of intercourse satisfaction of the IIEF. This was a negative correlation with sexual functioning as higher scores denote better functioning. These findings infer that a fair association of 'drinking years' with sexual dysfunction was observed. Therefore there as a fair association of drinking years with decreased Sexual drive, erectile dysfunction, problems of ejaculation, problem assessment of sexual functions and decreased overall satisfaction on the BSFI. On the IIEF increased years of drinking was associated with erectile dysfunction, enhanced problem of orgasmic function, decreased sexual desire and poor overall satisfaction. On the IPE, increased number of drinking years was associated with decreased sexual satisfaction, problems of control of ejaculation and distress related to premature ejaculation. No association was observed between the other variables and domains of sexual function of the BSFI, the IIEF or the IPE.

In our study $37(74 \%)$ subjects used tobacco (smoking or chewing) and no significant Correlation was found with any of the domains of sexual functioning. Similarly in the study by Simon Arackel and Vivek Benegal $87 \%$ of the subjects used tobacco and people with tobacco use were no more likely to have more sexual dysfunction than those without tobacco use (18).

\subsection{Serum Testosterone Levels}

Serum testosterone levels were assessed in all subjects of the study group. All the subjects had serum testosterone levels within the normal range except one subject who had increased serum testosterone levels.(Table 13). Testosterone ( $\mathrm{T}$ ) plays an essential role in normal male development as well as in the maintenance of many male characteristics. Animal studies demonstrated that nitric oxide $(\mathrm{NO})$ production is regulated by androgens which exert a direct effect on penile tissue to maintain erectile function. Androgen deficiency produces metabolic and structure imbalances in the corpus cavernosum, which results in veno occlusive dysfunction and ED. Studies in the past, have reported a decrement in serum testosterone levels after alcohol consumption This finding of normal serum testosterone also implies that none of our subjects had hypogonadism due to any other cause which could have contributed to sexual dysfunction.

\subsection{Ham A and Ham D Scores}

As depicted in table 14 the participants of the study group had score on HAM A and HAM D scales well below the cut off implying that none of them work significantly depressed or anxious. HAM A and HAM D were administered to the subjects to substantiate that there were no depression or anxiety symptoms in the study group. This was an essential exclusion criteria for the study group as depression and anxiety would have been confounding factors in the study. 


\section{Inferences and Explanation of These Results}

Multiple co-existing sexual dysfunctions seemed to be the norm in cases of alcohol dependence syndrome. The most common condition reported in our study was decreased sexual drive followed closely by erectile dysfunction. The chances of developing sexual dysfunctions appear to increase with increasing years of alcohol Consumed. Various explanations have been offered for sexual dysfunction in alcoholic patients. Higher level of alcohol intake may result in greater neurotoxic effects. It has been reported that heavy alcohol use may contribute to a reversible neuropathy, which is perhaps reversible on abstinence. Ethanol (ethyl alcohol) impairs spinal reflexes which causes both decreased sensation and decreased innervations for erection, but it has also been shown to decrease serum testosterone levels. Chronic heavy use of alcohol is also known to significantly alter gonadal hormones. There is also a significant population, which has psychogenic sexual dysfunction, which is likely in a situation of marital conflict, which commonly exists in the families of alcoholics or socio occupational stressors.

Erectile function is controlled by central nervous system (CNS) controls through dopaminergic (facilitative) and serotonergic system (inhibitory). Ejaculation is a complex Integration of action that occurs in the central and peripheral nervous system. Serotonin has inhibitory effects on ejaculation. Acute or chronic consumption of alcohol interferes differentially with transmission processes in the CNS, affecting many if not all, of the known neurotransmitter systems. Premature ejaculation may be due to associated alcohol related peripheral neuropathy.

\section{Summary and Conclusions}

1. Study had validated assumption that there is increased sexual dysfunction among alcohol dependent cases.

2. The prevalence of sexual dysfunction reported by the subjects was $72 \%$.

3. Mean scores of different types of sexual dysfunction was statistically significant compared to the control group.

4. There was an association observed between years of drinking and all domains of sexual function on all the scales.

5. There was no decrease or increase in serum testosterone levels in the study group.

6. This study stresses the need of addiction medicine specialists to note the possibility of sexual problems in their clients.

7. This information can be used in motivational counseling of heavy drinkers to provide impetus for change.

\section{Limitations of the Study}

The sample size of study was small. Spouses were not interviewed to find out any marital disharmony that could itself cause sexual dysfunction. The subjects were not reassessed after a period of abstinence to further determine if the sexual dysfunction persists. The quantity of alcohol consumed was not assessed and thus its association with the impairments in the domain of sexual function could not be evaluated. Only male patients were included in the study, therefore these finding do not apply to females.

\section{Intellectual Contribution of Author}

Study Concept: Dr Amitabh Saha, MD Psychiatry

\section{References}

[1] Avsthi A, Nehra N, Muthy RS. Sexual Disorders: A review of Indian Research.. Editor. Mental Health in India, Bangalore: People's Action for Mental Health, 2000.p.42-53

[2] Bancroft J. Sexual problems. In: Bancroft J. Human Sexuality and its Problems. $2^{\text {nd }}$ Ed. New York: Churchill Livingstone; 1983.p.360-411.

[3] Miller NS, Glod. MS. The human sexual response and alcohol and drugs. J Subst Abuse Treat. 1988;5(3):171-7

[4] Hamilton M. Development of a rating scale for primary depressive illness. Br J Soc Clin Psychol 1967, 6:278-296.

[5] Hamilton M. The assessment of anxiety states by rating. Br J med psycho 1959; 32:50-55

[6] Sadock VA. Normal human sexuality and sexual dysfunction. : Sadock BJ, Sadock VA, Ruiz P, editors. Kaplan \& Sadock's Comprehsive Textbook of Psychiatry, 9 ed. Philadelphia: Lippincott Williams and Wilkins; 2009,p 2027-2060.

[7] Oleary M.P, Fowler FJ, Lenderking R, Barber B, Sagnier PP, Guess HA, Barry MJ. A brief male sexual function for urology. 1995 Nov; 46 (5):697- 706.

[8] Rosen RC, Riley A Wagner G, Osterloh IH, Kirkpatrick J, Mishra A. The International index of erectile function (IIEF): a multidimensional scale for assessment of Erectile dysfunction. Urology 1997 Jun;49(6):822-30

[9] Althof. S, Rosen R, Symonds. T, Mundayat R, May Abraham L. Development and validation of a new questionnaire to assess sexual satisfaction, control and distress Associated with premature ejaculation. J Sex Med. 2006May; 3(3):465-75.

[10] Vijayasenan ME. Alcohol and sex. N Z Med J 1981;93:18-20

[11] Benegal V. India: alcohol and public health. Addiction 2005;100:1051-6

[12] Mandell W, Miller CM. Male sexual dysfunction as related to alcohol consumption: A pilot study. Alcohol Clinic Exp Res 1983;7:65-9

[13] Schiavi RC, Stimmel BB, Mandelli J, White D. Chronic alcoholism and male sexual Function. Am J Psychiatry 1995; 152:1045-51

[14] Jensen SB. Sexual function and dysfunction in younger marred alcoholics. A Competitive study. Acta Psychiatr Scand. 1984 Jun;69(6):543-9 
[15] Lewis RW, Fugl-Meyer KS, Bosch R, Fugl-Meyer AR, Laumann EO, Lizza E et al. Epidemiology/risk factors of sexual dysfunction. J Sex Med. 2004 Jul;1(1):35-9.

[16] Van Thiel DH, Gavaler J, Lester R. Ethanol inhibition of vitamin A metabolism in The testes: possible mechanism for sterility in alcoholics. Science. 1947 Dec 6; 186(4167):941-2.
[17] Fahrener. EM Sexual dysfunction in male alcohol addicts: prevalence and Treatment. Arch sex behav. 1987 Jun:16(3):247-57.

[18] Arackal BS, Benegal V. Prevalence of sexual dysfunction in male subjects with Alcohol dependence. Indian J Psychiatry. 2007 Apr; 49 (2):109-12. 